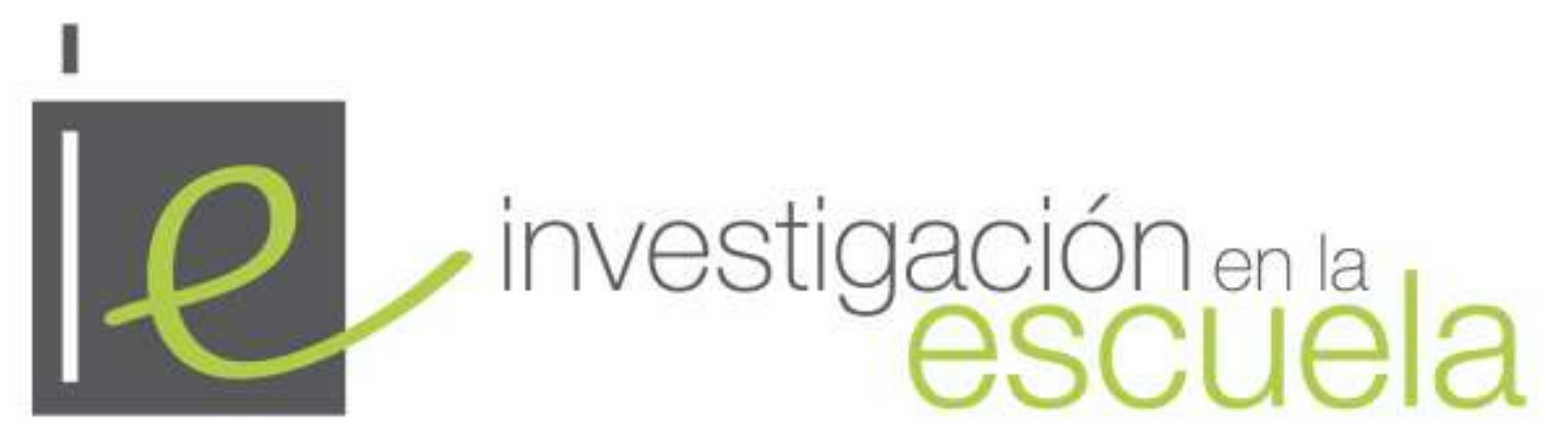

Revista internacional de investigación e innovación educativa

\title{
Valeurs en jeu, enjeux de valeurs. La justice dans des débats et discussions d'élèves
}

\author{
Philippe Haeberli \& François Audigier ${ }^{2}$ \\ Université de Genève \\ Suisse
}

Citación: Haeberli, P. \& Audigier, F. (2019). Valeurs en jeu, enjeux de valeurs. La justice dans des débats et discussions d'élèves. Investigación en la Escuela, 97, 32-46.

Résumé: L'article présente des travaux de recherche menés en Suisse romande entre 2005 et 2012. Ces travaux lient le concept de justice et des dispositifs d'échange organisés d'une part autour des conseils d'élèves dans le primaire, d'autre part sur l'étude d'une question de développement durable, la consommation de la viande suivie d'un débat. Avant de présenter quelques résultats ces travaux, nous explicitons l'intérêt du lien entre justice, école et éducation à la citoyenneté. Pour cela, nous faisons appel à diverses enquêtes menées en France, entre 1990 et 2010.

Mots-clés: "Acquisition de connaissances"; "justice"; "enseignement primaire"; "instruction civique".

Values at stake, value issues. Justice in student debates and discussions

Abstract: The article presents research conducted in French-speaking Switzerland between 2005 and 2012. This work links the concept of justice and exchange mechanisms organized around primary school student councils on the one hand, and on the other hand on the study of a sustainable development issue, meat consumption followed by a debate. Before presenting some of the results

\footnotetext{
${ }^{1}$ Citoyen genevois et suisse.
}

${ }^{2}$ Citoyen français et européen.

Página web: https://revistascientificas.us.es/index.php/IE

Facebook: / Revista Investigación en la Escuela IE

Artículo recibido: 30-07-2018

Revisiones recibidas: 15-01-2019

Aceptado: 24-02-2019 
of this work, we explain the importance of the link between justice, school and citizenship education. To do this, we use various surveys conducted in France between 1990 and 2010. Key words: "Learning"; "justice"; "primary education"; "civics".

Valores en juego, cuestiones de valor. La justicia en los debates y discusiones estudiantiles Resumen: El artículo presenta las investigaciones realizadas en la Suiza francófona entre 2005 y 2012. Este trabajo vincula el concepto de justicia y los mecanismos de intercambio organizados en torno a los consejos estudiantiles de la escuela primaria, por un lado, y el estudio de un tema de desarrollo sostenible, el consumo de carne seguido de un debate, por otro. Antes de presentar algunos de los resultados de este trabajo, explicamos la importancia del vínculo entre justicia, escuela y educación ciudadana. Para ello, utilizamos varias encuestas realizadas en Francia entre 1990 y 2010. Palabras clave: "Aprendizaje”; "justicia"; "enseñanza primaria"; "educación cívica".

\section{Le concept de justice}

La justice est un concept-valeur (cf. fin du chapitre précédent) particulièrement central pour la démocratie. Depuis la parution de l'ouvrage de J. Rawls, A Theory of Justice, au début des années 1970, les débats se sont largement diffusés dans les domaines éthique, politique, des sciences sociales et plus récemment environnementales avec la question de la justice intergénérationnelle (Gosseries \& Meyer, 2009). Il obéit à la logique de la construction exposée plus haut : en effet, ce sont les injustices notamment sociales auxquelles nous sommes confrontés qui nous aident, nous poussent à construire le concept de justice, à imaginer ce que devrait être une société juste. Il est également important de distinguer deux sens différents du terme justice. Dans un premier, la justice désigne la valeur qui nous sert à évaluer des situations, à juger en quoi tel ou tel cas est injuste ou non. Dans un second sens, la Justice (que nous écrivons avec une majuscule) désigne l'institution judiciaire, l'un des pouvoirs censés être indépendants des deux autres pouvoirs dans une démocratie. L'Ecole bien évidemment, n'échappe pas au questionnement de la justice, les élèves sont en premier lieu, les acteurs privilégiés pour expérimenter la justice de l'expérience scolaire.

\section{Le sentiment de justice des élèves}

Comme l'expérience sociale, l'expérience scolaire est aussi porteuse d'injustices. Les élèves y sont évidemment sensibles. Dès les années 1990, des chercheurs se sont intéressés au sentiment de justice des élèves (Dubet, 1999 ; Duru-Bellat \& Meuret, 2009 ; Merle, 2005). L’intérêt de leurs travaux est double : 1) montrer que les injustices perçues par les élèves structurent les comportements et ont des conséquences multiples dans le champ scolaire, 2) montrer l'étendue des injustices dénoncées par les élèves et la grande sensibilité de ceux-ci aux injustices. A partir d'entretiens approfondis avec des élèves du Lycée ${ }^{3}, F$. Dubet met à jour trois principes qui structurent fortement le discours de ceux-ci : le mérite, l'égalité, le respect. Le premier principe se rapporte à l'individu et concerne avant tout le domaine de l'évaluation qui doit répondre selon les élèves au principe de l'équivalence entre contribution et rétribution; le deuxième se rapporte au groupe classe, au souci de son maintien, de sa cohésion ; enfin, le troisième renvoie au respect de la personne de chacun et de sa dignité, il fait écho, a contrario, au thème du mépris mis en évidence comme facteur dominant le discours des lycéens dans leur rapport aux professeurs (Dubet, 1991).

Certes, dans la classe, les textes réglementant l'action de l'enseignant existent, mais ils ne sont pas toujours appliqués dans la pratique. Les enseignants outrepassent parfois le pouvoir qu'il leur est

\footnotetext{
${ }^{3}$ Établissement d'enseignement accueillant la deuxième partie des études secondaires, en France, trois années terminées par le baccalauréat.
} 
conféré, ce qui peut les amener à tomber dans l'arbitraire. Les élèves reçoivent naturellement très mal ces actes et/ou paroles du Prince. Deux enquêtes menées en France appuient ce constat. Le sociologue français Pierre Merle (2002) montre que l'humiliation des élèves est pratiquée de manière consciente ou non par certains enseignants ; elle est utilisée comme instrument non-réglementaire de pacification de la classe. Ces pratiques, qui recouvrent aussi bien le rabaissement scolaire lié au statut d'élève que l'injure liée à la personne, visent en général un élève sans qu'une faute scolaire ait été clairement identifiée. Une enquête sur la punition scolaire menée par un autre sociologue français Eric Debarbieux (1999) révèle que plus de deux tiers des sanctions édictées par les enseignants (par exemple, pensum, travaux supplémentaires, exclusion de l'élève de la classe seul) sont proscrites par les textes officiels.

Autre point de focalisation des injustices scolaires, le problème de la réciprocité. Un jugement est estimé juste quand l'enseignant, les adultes en général, s'y soumettent au même titre que les élèves : le maitre doit s'imposer les règles qu'ils imposent aux élèves, il doit être respectable, s'il veut être respecté. Il doit être ponctuel, courtois et juste, comme les élèves doivent l'être. L'enquête de Dubet (1999) souligne également le fait que les élèves ont beaucoup de peine à comprendre et à admettre de telles inégalités de traitement. Enfin, un dernier aspect important du jugement de justice toujours relevé par Dubet (1999) porte sur la difficulté dans la montée en généralité des critiques. Il est très rare de rencontrer un discours d'élève dans lequel est évoquée la possibilité d'une autre école. Il y a une absence de critique de nature sociale de l'institution scolaire ou plus largement du système, dans les discours des collégiens interrogés. À aucun moment, les élèves ne relèvent, par exemple, des différences de traitement telles que « les enseignants choisissent l'égalité pour ce qui les concerne (carrières à l'ancienneté) et optent pour le mérite pour juger les élèves ». Même si les enquêtes sont rares et, donc, une certaine prudence de mise, les élèves interrogés semblent avoir beaucoup de peine à penser leur situation autrement que dans la centration sur la relation maître/élève à l’intérieur de la classe de cours.

\section{La justice du système scolaire}

Enjeu majeur du monde d'aujourd'hui, le thème de la justice soulève la question des inégalités qui ne cessent d'augmenter depuis quelques décennies. En écho à la montée de ce thème dans le monde, les débats contemporains autour de la justice sont intenses. Ils posent la justice comme valeur cardinale vers laquelle devrait tendre tout État démocratique. Nous ne reprenons pas ici ces débats. Mais il est une question qui concerne notre propos : en tant qu'institution, l'École l'École publique s'entend -, n'a-t-elle pas été instituée dans une perspective de justice ? L'École estelle juste dans la manière dont elle produit des apprentissages et délivre des diplômes qui garantissent en principe, un rang social. Depuis la massification des effectifs scolaires observée dans les années 1960 en Europe et en Amérique du Nord, la théorie de l'égalité des chances est une des manières de compenser les inégalités de réussite liées à l'origine sociale (Coleman, 1966) aux EtatsUnis, Bourdieu \& Passeron (1970) en France. Pour que les plus talentueux ne pâtissent pas d'un handicap lié à l'babitus pour reprendre le terme de Bourdieu, certaines inégalités de traitement se trouvent justifiées.

L'action éducative est, par exemple, orientée vers une discrimination positive par l'octroi de moyens supplémentaires pour les écoles des zones socialement défavorisées. L'expérience des zones prioritaires d'éducation en France ou des réseaux d'enseignement prioritaire à Genève, en sont un exemple. Toujours en s'appuyant indirectement sur la théorie de Rawls (1971), le questionnement sur l'école à partir du concept de justice s'est également fait en termes d'équité. Certaines inégalités sont tolérées parce qu'elles ne sont pas jugées assez injustes pour être intolérables. Ainsi, certaines inégalités générées par les systèmes éducatifs sont d'autant plus injustes que leurs conséquences sur la vie future des élèves sont importantes, qu'elles sont attribuées au fonctionnement du système 
éducatif plutôt qu'au contexte social et culturel, qu'elles se réalisent au détriment des défavorisés et qu'elles affectent fortement le jugement que les citoyens ou usagers portent sur la justice du système éducatif (confiance dans l'institution).

\section{Le récit de justice}

Pour les individus mais également les acteurs collectifs, raconter le monde, en faire un récit est un moyen privilégié pour saisir l'intelligibilité et le sens de celui-ci (Ricoeur, 1983). Dans la perspective générale du récit comme appréhension du devenir et de la conduite du genre humain, la dimension temporelle de l'expérience est consubstantielle au devenir humain : "Dire l'expérience humaine, c'est toujours aussi la raconter ». Pour Ricoeur, la notion de récit est ancrée dans des considérations philosophiques et morales possédant une dimension normative. Le récit couple une posture descriptive à une posture éthique: les sujets sociaux font empiriquement l'expérience de leur propre existence au travers de narrations ou d'assemblage de petits récits mais les sujets se construisent également par la pratique narrative, la mise en intrigue ${ }^{4}$ de leur propre expérience qui se traduit par une forme d'appréciation du « réel ». La mise en récit de l'expérience n’échappe donc pas à la dimension morale, elle ne peut faire l'économie d'une forme de jugement moral : le récit n'est « jamais éthiquement neutre et s'avère le premier laboratoire de jugement moral » (Ricoeur, 1990, p. 167).

Pour le psychologue J. Bruner, il existe ainsi un récit collectif qui fonde la démocratie, le récit de justice issu de la manière dont la Justice, les tribunaux rendent justice. Ce récit contraint la manière dont des situations sociales vécues comme injustes ou comme ayant causé du tort par les personnes en fonction de normes juridiques mais aussi par le biais de procédés d'assainissement (Bruner, 2002). Bruner caractérise ce récit qui amène au jugement de la manière suivante : le récit de justice est «intrinsèquement rhétorique dans sa forme et légitimement ouvert au doute »; il est caractérisé par un caractère personnel, motivé par une position « partisane » et directement concernée par le résultat. Narratif dans sa structure, partisan et personnel, le récit de justice est assaini par l'esprit contradictoire qui le guide ; il relève en fin de compte de notre « foi dans les vertus de la confrontation » qui « doit nous permettre d'aller au fond des choses» (Bruner, 2002, p. 39). Le récit de justice est au fond et pour dire les choses simplement, une transposition de la vertu attaché au débat dans une démocratie.

Dans la suite de ce texte, nous utilisons le récit de justice comme analyseur des constats faits dans deux recherches menées à Genève et dans trois autres cantons francophones de Suisse romande. Avant de présenter les résultats, nous évoquons la méthodologie et le terrain de chacune des recherches. La première est une recherche doctorale (Haeberli, 2009) dont l'objet d'étude est le conseil d'élèves. Le terrain d'enquête est constitué de deux écoles primaires. La population enquêtée vient principalement du cycle moyen du primaire genevois (3P-6P) ce qui correspond aux quatre dernières années de l'école primaire genevoise ${ }^{5}$. Le matériau analysé comprend des séances de conseil enregistrées en vidéo et observées, des entretiens qui se sont déroulés par groupe de trois élèves, issus de sept classes différentes et des entretiens avec les enseignants. La deuxième recherche dont le principe de base le détour / retour est présenté dans le chapitre précédent, a été menée par une équipe de recherche en didactiques et épistémologie des sciences sociales (ERDESS), réunissant des chercheurs et formateurs de différentes institutions de Suisse romande. La séquence d'enseignement-apprentissage impliquant l'étude de situations sociales et des débats concernant des questions sociales vives à propos de l'alimentation, a été mise en œuvre dans une quinzaine de classes de la fin du primaire et du secondaire I.

\footnotetext{
${ }^{4}$ Qui se charge de faire la « synthèse de l'hétérogène » selon l'expression de Ricoeur.

${ }^{5}$ Les élèves participant aux entretiens sont ainsi âgés de sept/huit à onze/douze ans.
} 


\section{Quel récit de justice dans les discussions en conseil d'élèves?}

La pratique des conseils d'élèves donne à ces-derniers la possibilité de délibérer sur des problèmes ou des conflits liés à la vie scolaire. Le conseil endosse dès lors une fonction judiciaire et/ou une fonction exécutive. Il est dès lors intéressant de se pencher sur la manière dont ces fonctions sont distinguées et comment les décisions sont prises sous l'angle de la justice et notamment sous l'angle de la confrontation des points de vue.

\section{Une pratique coopérative}

Le conseil d'élèves en tant que lieu d'échanges et d'interactions sociales entre membres de la classe, réunissant maître et élèves, se présente comme un lieu privilégié de la co-construction d'un cadre de contraintes morales pour l'exercice de la justice dans la classe et, plus largement pour organiser la vie scolaire en fonction des principes de la justice. L'Éducation nouvelle et les pédagogies nouvelles comptent parmi les premiers mouvements importants dans l'histoire de l'éducation à tenter d'organiser l'expérience scolaire en fonction des principes de la régulation juridico-politique (Prairat, 2003).

Sans entrer dans le détail du conseil coopératif tel que l'entendait Freinet (Freinet, 1971) ou l'entendent aujourd'hui les tenants de la Pédagogie Institutionnelle, héritière de ces pratiques fortement liées aux mouvements d'Éducation nouvelle, nous retenons que ce lieu est pensé comme lieu de discussion, d'échanges mais aussi et surtout comme moyen de responsabiliser les élèves par la résolution de problèmes et/ou de conflits, que ceux-ci et ceux-là soient reliés au travail et à l'organisation scolaire. Les différents rôles assumés par les élèves - président de séance, distributeur de parole, secrétaire consignant les problèmes et les décisions, etc.- participent de cet apprentissage de la responsabilité mais il n'est pas confiné à cet aspect procédural. Il s'agit également de faire fonctionner un milieu coopératif et de coordonner divers dispositifs pédagogiques (imprimerie, correspondance, production de texte libre, etc.) (Vergnioux, 2005) tout cela à des fins de formation d'un individu libre apte à perpétuer et inventer des formes démocratiques de société (Freinet, 1960). Proche de la philosophie du learning by doing de Dewey (1955), Freinet pense que la citoyenneté comme la morale «ne s'enseigne pas mais se pratique ».

\section{Les pratiques à Genève}

À l'école primaire à Genève, la diffusion des conseils d'élèves s'est faite au niveau de la classe et de l'école. Elle a été importante dans les années 1990 et 2000. Elle est en grande partie le fait d'un entraînement entre collègues et s'est déroulée en l'absence de toute orientation curriculaire et de tout cadre de formation initiale. Dans le discours enseignant, deux ordres de raisons sont principalement avancés à leur mise en œuvre (Laplace, 2002). Des raisons d'ordre pratique qui relèvent de la gestion de la classe; des raisons d'ordre plus didactique assimilant le conseil de classe à une instance démocratique dont l'expérience donne l'occasion aux élèves de s’initier aux formes démocratiques du fonctionnement social.

\section{Un récit de justice très largement scolarisé}

Le récit de justice des conseils observés à Genève sont déterminés par des éléments de la forme scolaire (Vincent, Lahire \& Thin, 1993), par la manière dont l'école organise ou souhaite organiser les relations sociales au sein de la classe.

Dans leur fonctionnement global, les conseils d'élèves à Genève sont composés au minimum de quatre responsabilités assumées par des élèves : un président qui lit les mots, mène les débats, organise les votes, etc. ; un donneur de parole qui est chargé de distribuer la parole pour permettre l'échange ; un ou deux gardien(s) du silence ou $\operatorname{arbitre(s)~qui~note(nt)~les~transgressions~aux~règles~de~}$ 
l'échange ; enfin, un secrétaire qui note les décisions prises. Les seules responsabilités auxquelles il est donné du pouvoir sous forme de sanctions qui peuvent aller jusqu'à l'exclusion de l'élève du conseil, sont celles de gardien du silence ou d'arbitre, c'est-à-dire les responsabilités chargées de faire respecter et intégrer les règles de l'échange : on lève la main pour parler et on attend d'être appelé pour parler. L'intégration de la coutume scolaire semble bien être la finalité centrale de la pratique des conseils d'élèves.

Les petits conflits du quotidien de la classe, de la vie scolaire, ce que l'on peut ranger sous le terme générique d'« incivilités », sont majoritairement les objets traités dans les conseils de classe. Dans le jargon adopté dans les conseils, les mots majoritairement traités correspondent à des critiques qu'un ou plusieurs élèves adressent à un ou plusieurs autres élèves de la classe. La légitimité des critiques s'appuie essentiellement sur les normes scolaires de comportement à propos des relations entre pairs (" pousser», «taper», « bagarrer», etc.), le travail dans la classe (" déranger », " parler sans arrêt », « ne pas écouter », « se lever », etc.) et les règles de la civilité (« se moquer », « dire des gros mots », « être impoli »).

Parmi les solutions apportées aux critiques, la demande d'effort est majoritaire dans les séances observées. On demande à l'élève incriminé de "faire un effort », d'« arrêter petit à petit », d'" essayer de changer » son comportement ou son attitude. Le mode de résolution est la recherche de l'accord des parties et non la décision par le groupe ; celle-ci est toutefois scolarisée et débouche sur une forme coutumière de résolution: l'accord des intéressés est le plus souvent tacite, c'est l'accumulation des propositions qui amène le secrétaire à retenir et noter une solution dans le cahier du conseil. Dans trois conseils, l'appel à l'effort atteint des proportions telles (environ deux tiers des solutions aux critiques) que le recours à l'effort devient lui-même un objet routinier et coutumier. Dans l'un des rares mots déplorant le manque de solidarité dans la classe, le secrétaire accompagne la solution routinière du commentaire suivant :

Un élève: C'était quoi, la solution?

Le secrétaire : Ben, EF, comme ça... Effort, comme ça tout le monde sera mieux et puis il y aura moins d'insultes et tout ça, tout rentrera dans l'ordre. (6P).

La norme de l'effort est ainsi érigée en principe régulateur de la vie de la classe ; du point de la justice, il renvoie directement au principe méritocratique dominant l'Ecole (Crahay, 2000): appliqué au domaine des relations sociales, les efforts permettent d'atteindre un monde plus juste, plus harmonieux. Du point de vue de la justice, le récit des conseils genevois écarte toute idée de confrontation ou de volonté d'aller au fond des choses. Ceci renvoie plus largement à la difficulté à l'école d'aborder les différences de point de vue ou les savoirs instables.

\section{Des jugements de justice complexes}

Dans la même recherche doctorale (Haeberli, 2009), lors d'entretiens avec les élèves, le récit spontané de justice d'une majorité de groupes d'élèves interviewés porte sur le risque d'arbitraire lié au favoritisme découlant de l'exercice des responsabilités comportant un pouvoir effectif. Les injustices

vécues par les élèves, aussi bien dans les petits degrés que dans les plus grands, portent sur les effets des liens d'amitié ou d'inimitiés entre les élèves sur l'exercice de la responsabilité:

Le donneur de parole, il peut être plus ami avec l'accusé et il aime pas du tout l'autre, donc il va pas lui donner la parole. (Michel) 
Je sais pas, par exemple Marc qui parle, parce que pratiquement personne l'aime, je lui donnerai un carton et puis mes copines elles sont peut-être en train de se marrer, puis je leur donnerai pas. (Corinne)

Tu mets pas de coches à tes copains et ceux que tu n'aimes pas t'en mets plus. (Alain).

Dans une première approche, la confusion entre le registre de l'amitié et celui des obligations ou des devoirs liés à la responsabilité structure les récits de justice des élèves. Ceci s'explique notamment par la force des liens affectifs chez des jeunes élèves et dans un groupe qui reste très stable tout au long de l'année. Toutefois, l’identification de la confusion des registres dans les récits de justice ne débouche pas nécessairement sur une critique de justice, sur des jugements de justice.

Trois attitudes sont à distinguer dans les groupes examinés : une partie des élèves reste au constat d'une confusion sans articuler celle-ci à l'énoncé d'une évaluation; une deuxième attitude, privilégiée par les élèves des plus petits degrés, consiste à faire appel au pouvoir du maitre pour encadrer les responsables et contrôler le comportement de ceux-ci « Parce que si la prof elle voit, elle dira sûrement qu'il faut donner la parole aux autres, quoi » (Kevin) ou encore demander la (re) délégation de l'attribution au maitre : «les avertissements dans le conseil, faut que ce soit la prof » (Alice). Enfin, une partie des élèves formule des jugements de justice à la suite de l'identification de la confusion des registres. Pour ces derniers qui appartiennent aux plus grands degrés, l'équité dans la distribution de la parole, la possibilité de priver un individu du droit à la parole, du droit de s'exprimer, sont mentionnées à maintes reprises comme injustes:

Il faut quand même que ce soit juste pour que tout le monde puisse parler. (Michel)

Moi je mettais pas de carton parce qu'y a chacun qui doit avoir son opinion. (Sophie)

Le donneur de parole, il ne peut pas parler sans lever la main, il va attendre d'interroger les personnes qui lèvent la main car sinon il aurait tous les droits. (Marie).

Ainsi, dans les échanges en petits groupes, les récits d'une partie des élèves soulèvent, le plus souvent spontanément, des questions de justice engendrées par l'exercice des responsabilités dotées de pouvoir effectif. Dans les récits des injustices vécues, les élèves manient, dans l'argumentation et la justification de leur sentiment d'injustice, des notions appartenant au domaine du droit et de la philosophie politique comme, par exemple, l'équité dans la parole, l'égalité de traitement, le refus de l'arbitraire. Ces compétences de justice exercées par les élèves relèvent, en creux, la cruelle absence de référence au droit dans la pratique des conseils. En effet, une règle démocratique de base veut que tous les pouvoirs doivent se soumettre aux règles qu'ils produisent. C'est bien l'absence d'une telle règle ou d'un tel contrôle que les élèves dénoncent à travers leur sentiment d'injustice.

Les compétences que les élèves mettent en œuvre en entretien, devrait pouvoir être exercé en conseil. Si la plupart des individus ne sont pas forcément en mesure de dire très clairement ce qui est juste et ce que devrait être un monde juste, tous disent facilement ce qui leur semble injuste et pourquoi. A la question « pourquoi pensez-vous que la situation ou la conduite que vous venez de 
dénoncer est injuste ?", chacun est souvent en mesure de développer des arguments de principe et de se conduire ainsi en «philosophe » (Boltanski, 1990). C'est en ce sens même que le philosophe américain Michaël Walzer a défendu l'idée que la critique sociale est une activité sociale " naturelle » (1990). Encadrée par les principes du droit et d'un travail de conceptualisation, ce type de critique sur la vie scolaire, permettrait de développer d'évidentes compétences citoyennes.

\section{Quel récit de justice lors de débats autour d'une question socialement vive?}

Dans la seconde recherche que nous présentons, il y a une réalité sociale à comprendre et qui appelle décisions et actions. Cette réalité n'est pas disciplinaire, les décisions à prendre non plus. Mais l'étudier, l'analyser, la raisonner, requièrent des outils et des savoirs disciplinaires. C'est la phase du détour au cours de laquelle sont introduits et construits des savoirs et des outils disciplinaires. Puis, il s'agit de mobiliser ces savoirs dans une situation différente, c'est la phase du retour. Pour introduire et étudier cette phase de retour, nous avons choisi de placer les élèves dans des situations de débat (Audigier, Fink, Freudiger \& Haeberli, 2011).

Ainsi, en 2010 et 2011, des élèves du primaire et du secondaire I de quatre cantons suisses romands ont été placés dans un dispositif de débat. Avant de débattre, ils ont travaillé sur un thème d'actualité, les ressources alimentaires, plus particulièrement la viande. Sur ce thème, les élèves du primaire ont débattu d'une pétition d'un parti politique visant à instaurer une journée sans viande dans les cantines scolaires, ceux du secondaire, du droit qu'aurait l'ensemble des humains à consommer de la viande 'comme nous'. Ces débats ont été 'ouverts', c'est-à-dire qu'une fois la question posée et éventuellement précisée et expliquée, les élèves intervenaient librement pour peu qu'ils demandent la parole ; aucune autre consigne ne leur était donnée ou rappelée que celle d'exprimer et de justifier leur point de vue.

Nous présentons deux extraits de débats de classe issus des transcriptions des enregistrements vidéo réalisés lors des débats, transcriptions faites avec le logiciel Transana. Elles sont ici 'nettoyées', au sens où nous avons éliminé les hésitations, les répétitions, etc., pour ne conserver que ce qui s'énonce comme l'essentiel du point de vue de l'élève. Les 'gras' sont de notre fait.

\section{Extrait 1 : un même droit pour tous ?}

Jérôme : Ben, en fait faudrait quand même que tout le monde en mange parce que ceux qui ont pas le droit de manger de la viande c'est quand même méchant pour eux. Parce que, y'a dans des pays ils mangent plus de viande que d'autres. Donc c'est un peu injuste, pour ceux qui mangent moins de viande.

Enseignant: Oui, mais que faire : produire plus ? Consommer moins?

Jules : Moi, je pense que tout le monde a le droit de manger de la viande, mais on va pas pouvoir parce que on va déforester et y aura un impact écologique à cause du CO2 du aux transports et la qualité de la viande ne sera pas la même. Donc, ça va pas être vraiment possible pour tout le monde.

Enseignant : Des réactions par rapport à ça? ...

Alice: Bah moi je pense qu'il faudrait réduire la quantité de viande qu'on mange parce que c'est beaucoup trop par rapport à nos jours et pis c'est surtout pour l'écologie parce que avec les transports ça fait y aura de plus en plus de C02 et pis y aura de plus en plus de terrains 
pour élever le bétail et ça va être beaucoup trop exagéré pour juste l'égoïsme d'avoir son steak tous les jours.

L'affirmation de l'égalité des droits fonde la prise de position. L'inégalité vaut ici injustice. Augmenter la production a des effets négatifs : la déforestation et ses conséquences; la distance entre les zones de production et celles de consommation. La réalité rend l'égalité de droit impossible à garantir. Il vaut mieux baisser la consommation ce qui implique aussi de lutter contre notre égoïsme. Le droit, les valeurs, le possible sont étroitement mêlés.

Les élèves mêlent les savoirs étudiés avec ce qu'ils connaissent déjà du thème et de la situation, et les interprétations avec lesquelles ils appréhendent le monde et les activités humaines. Ainsi, par exemple, sans qu'il ait été systématiquement enseigné, le rapport entre offre et demande et son effet sur les prix intervient souvent comme une clé de lecture des conséquences d'abord monétaires puis sociales. Si la demande baisse, les producteurs paysans seront touchés. Si l'offre de viande augmente, les prix vont diminuer; mais si la demande augmente plus vite que l'offre, les prix vont augmenter et la viande sera réservée aux plus riches.

Nous reprenons ici le terme d'obstacle introduit par Martinand avec le concept d'objectifobstacle (1984) et le détourne pour relever deux obstacles que l'on peut ou non transformer en objectif : ignorance-obstacle ; certitude-obstacle. Par la première association, nous désignons le fait que les élèves ont souvent des difficultés à prolonger leur raisonnement car ils n’ont pas de connaissance pour le faire ; par exemple, ils ignorent les pouvoirs effectifs des différents acteurs, en particulier les compétences des institutions politiques. Un exemple de certitude-obstacle exprimée dans les extraits reproduits ci-dessus concerne le poids du transport comme facteur principal de pollution. Or, les conditions de production ont un impact beaucoup plus important que le transport même au long cours. On rejoint ici aussi la question des stéréotypes et des informations très largement diffusées dans de nombreux médias.

\section{Extrait 2}

Anna: Mais faut pas penser qu'à soi, faut aussi penser aux autres, parce qu'on est pas tout seul sur terre et y a tout le monde qui a des envies mais on peut pas que répondre que à nos envies, faut aussi penser à celles des autres.

Enseignant: Y avait pas mal de mains qui se levaient là?

Manon: Bah moi je pense que si vous dites ça, vous êtes égoïste et que si heu vous étiez né dans une famille heu dans un pays pas très développé vous auriez pensé autre chose.

Marco: Heu moi je dis que,bah chui un petit peu comme heu Monsieur Dialow là qui avait dit que, que faut qu'on préserve la planète mais en même temps, on, faut qu'on mange de la viande, justement pas, pas tous les jours mais p't'être une fois par semaine ou deux, comme ça. 
Marie: Bah moi je pense qu'il faudrait réduire la quantité de viande qu'on mange parce que c'est beaucoup trop par rapport à nos jours et pis heu c'est, c'est surtout pour l'écologie parce que heu, avec, avec les transports que ça, que ça fait ben y aura de plus en plus de C02 et pis heu, et pis heu y aura de plus en plus de terrains pour faire heu, pour faire, pour élever le bétail et heu ça va être heu, beaucoup trop exagéré pour heu juste avoir heu, pour juste l'égoïsme d'avoir son steak tous les jours.

Catherine: Bah moi je pense que il faut savoir aussi un peu se priver des, de plaisirs parce que ben là heu peut-être heu pour vous ça va, ça va, ça va rien faire directement là mais dans les générations d'après ben il va y avoir des, des changements et pis ben ils sont pas vraiment content en fait, fin. (...)

Coralie: Heu en même temps heu, quand vous dites ça comme ça, heu c'est égoïste et tout mais enfin on mange et on mange de la viande aussi, on, enfin, à chaque fois qu'on va manger un, fin heu tout le monde heu ch, ch, chai pas si vous quand vous mangez de la viande vous allez vous dire tout, que ça va dérégler la nature et tout fin (...). Fin si y a quelqu'un qui se dit ça heu bah c'est vraiment, c'est bien mais...en même temps c'est égö̈ste mais en même temps heu on, on le fait un peu tous. (...)

Marie: Moi je trouve que la viande c'est quand même bon pour la santé alors si on pouvait juste heu réduire sa, la consommation et pas heu tout à fait l'interdire comme heu dit Monsieur Lopez, donc heu... (...)

Catherine: Bah en pensant ça, on pense un peu qu'à soi-même, il faut penser aux autres, heu, heu aux générations qui vont venir après mais que ce sera pas la même chose pour eux, ils auront, si on continue comme ça ils auront pu, plus la possibilité de, bah d'en manger.

Dans les propos des premiers intervenants, un conflit met en opposition des valeurs d'altruisme et de solidarité avec son prochain ou avec la nature et la planète, et la condamnation de l'égoïsme de celui ou celle qui ne serait pas garante de ces valeurs. A nouveau, on peut souligner la force du rejet de la pollution, et surtout celle provoquée par les transports.

Une élève en particulier, Catherine, œuvre tant bien que mal à ouvrir vers un débat plus conflictuel ; elle verse au débat, à la fin de la première partie de l'extrait, la question de la solidarité avec les futures générations et se positionne clairement en faveur de la valeur à accorder à l'avenir. Pour argumenter ses propos et raisonner l'action qu'elle propose, elle mobilise la ressource cognitive de l'échelle temporelle, en maniant le présent et l'avenir.

C'est plus tard que deux élèves (Coralie et Marie) introduiront dans la discussion un doute sur les positions consensuelles exprimées précédemment pas leurs camarades. La première, Coralie, remet en question l'accord qui semblait s'être établi entre les interventions précédentes de ses camarades et n'hésite pas à contrer la condamnation d'égoïsme. Elle souligne la distance qui peut exister entre l'affirmation de beaux principes et la traduction effective de ces principes dans les actions réelles. Marie complète la remise en question lancée par Coralie en ajoutant la valeur de la santé humaine. Ces deux élèves défendent leurs points de vue de consommateur en faisant valoir un 
certain individualisme, mais un individualisme « réaliste » et non égoïste, en faveur de la santé humaine.

Il semble toutefois que le doute et la distance apportée par ces deux élèves n'aient pas réussi à faire changer Catherine de position éthique, puisque par la suite, elle intervient sans tenir compte de la valeur de santé avancée par Marie. Elle adopte à nouveau une position étique de type déontologique en focalisant à nouveau son argumentation sur des valeurs d'altruisme et de solidarité avec les générations futures.

De manière générale, les élèves expriment l'imbrication étroite entre les éléments factuels et les valeurs sur lesquelles ils appuient leur point de vue. Parmi ces valeurs, les couples égalité/inégalité et liberté/contrainte sont les plus fréquents. Le premier est lié à justice tandis que le second appelle fréquemment l'efficacité. Celle-ci met aussi souvent les élèves en situation de dilemme, la liberté est prioritaire, mais les humains étant ce qu'ils sont, l'efficacité de telle ou telle mesure demande que l'on agisse parfois avec la contrainte, notamment celle de la loi et donc de l'autorité publique. Dans l'usage des valeurs, on distinguera des valeurs liées à la situation et au point de vue exprimé à son propos et des valeurs d'action liées à ce qu'il convient de faire. Ainsi, en appeler à l'égalité fonde la prise de position et l'efficacité sera appelée avec l'action. (Haeberli, Freudiger \& Audigier, 2013).

\section{Diversité et rôle de la question du débat}

La question posée et débattue joue un rôle important dans la dynamique du débat. La différence entre celle posée dans le primaire et celle posée dans le secondaire souligne une question d'apprentissage aussi peu étudiée dans nos didactiques qu'importante et constamment présente : la relation entre le singulier et le général. Les modes de raisonnement et l'usage des exemples ne sont pas les mêmes. Ainsi, le projet de loi des Verts mis en débat dans le primaire était 'local', singulier, au sens où il concernait directement les élèves. Dans ce cas, ceux-ci partent de leur situation, de leurs goûts, imaginent les conséquences, pour eux, de la mise en œuvre d'une telle restriction. Assez vite la réflexion s'ouvre aux dimensions plus générales liées à la recherche des raisons et effets d'une telle proposition elle-même liée à l'augmentation de la consommation de la viande dans le monde. Ils montent en généralité. Mais, ils vont puiser d'abord dans leur expérience, dans les conséquences pour eux, les arguments, exemples, contre-propositions, montrant qu'ici l'action personnelle est la première référence. En quelque sorte à l'inverse, lorsque la question porte directement sur le droit pour tous, ce sont d'abord des valeurs générales d'égalité et de justice qui sont exprimées et soustendent les prises de position favorables au droit. Lorsque des limites sont mises à l'exercice du droit, ils vont d'abord utiliser des facteurs, des réalités 'générales'... 'la' pollution, 'les' transports, 'le' système industriel, 'la' demande, etc.

Le dernier point que nous soulevons est celui de la diversité. Durant les débats, les élèves expriment des positions diverses. Cela est attendu et souhaité. D’une part les questions posées appellent des réponses plurielles, d'autre part le principe du débat démocratique pose cette diversité comme à la fois un fait et une nécessité. Les différentes données recueillies au cours de la recherche mettent en évidence plusieurs positionnements : les défenseurs de la nature ; les fatalistes qui considèrent que l'on ne peut pas faire grand-chose ; les confiants dans la science ; les convaincus de la nécessité d'un changement profond dans nos modes de vie ; ceux qui se fixent d'abord sur la recherche d'une consommation raisonnée ; ceux qui n'ont pas l'intention de modifier quoi que ce soit dans leur manière de vivre. Cette diversité rejoint celle que l'on observe dans l'ensemble de la population. Toutefois, les attentes relatives à l'EDD impliquent d'interroger cette diversité. Celle-ci est d'abord un constat, un fait d'enquête. Mais, comme pour les valeurs, y a-t-il des points de vue inacceptables, au sens où leur pertinence, voire leur légitimité, peuvent être mises en question? 


\section{Un necessaire elargissement}

Nous avons évoqué des résultats de recherche qui portent sur l'étude de dispositifs aptes à mettre les élèves en mesure de construire un récit de justice pour régler des problèmes et/ou des conflits liés à la vie scolaire ou contenant des dilemmes appelant des choix de valeurs sur des questions socialement vives, comme le réchauffement climatique et la consommation de viande dans le monde. Si dans le premier cas, l'observation des pratiques de conseil d'élèves dans les écoles primaires à Genève fait apparaître un récit de justice très scolarisé, orienté vers le maintien de l'ordre et la pacification de l'espace scolaire, évitant tout débat autour de la justice, les mêmes élèves pris à part en entretien, portent un intérêt à l'injustice des situations scolaires, en débattent et forment même pour certains des jugements pertinents reposant sur le concept d'égalité complexe (Walzer, 1998). Dans les situations de débat sur les QSV, les élèves qui sont aussi en partie des élèves de secondaire, identifient des dilemmes de valeurs sous-jacents aux enjeux sociaux et éthiques liés aux questions qui orientent les débats proposés. Il y a certes une question d'âge à considérer et des variations liées à la question qui oriente les débats mais le fait d'élargir la zone de questionnement et recherche de solution au-delà de questions de vie scolaire, concourent à enrichir le récit de justice produit par les débats entre élèves.

Ces observations sont d'autant plus importantes que, depuis quelque temps, le « débat » est devenu une sorte de norme pédagogique qui rassemble une pluralité de dispositifs, d'intentions, de fonctionnements, qu'il convient de clarifier et d'expliciter (Audigier, 2016). Enfin, elles ouvrent aussi l'immense domaine d'analyse et de réflexion portant sur la part des émotions, des affects, des croyances, dans la construction des jugements de chacun. De plus en plus d'auteurs considèrent que les unes et les autres fondent en grande partie ces derniers (par exemple : Damasio, 2018 ; Lordon, 2013 ; Walzer, 2003).

\section{Suspension : quelques inquietudes et orientations}

A partir d'un examen de l'éducation à la citoyenneté (voir chapitre précédent) et de résultats d'enquêtes menés auprès des élèves, nous avons rencontré de nombreux questionnements impliquant analyse critique et approfondissement. Plusieurs directions de réflexion prolongent notre examen. Parmi celles-ci les unes renvoient aux conceptions des apprentissages, d'autres sont plus directement liées à la vision du monde, aux imaginaires, à la Weltanschaunng que l'on choisit et décide de transmettre. Dans les premières, nous rangeons les questionnements relatifs à la place de l'expérience. Nous avons rappelé les critiques dont une éducation politique trop formelle a été et est toujours l'objet et l'importance des courants qui privilégient les mises en situation. Les critiques contre le formalisme ne sont en aucun cas une validation du rôle de l'expérience. Là encore nous sommes dans deux manières de comprendre et d'organiser les apprentissages dont l'un n'est pas l'envers de l'autre. Ainsi, l'expérience n'est pas nécessairement source d'apprentissage, l'expérience n'est pas transparente à l'acteur. Cela implique de mettre les savoirs et les expériences dans une position complémentaire et dialogique et non dans une sorte de compétition au choix obligé. Aucune conscience démocratique ne se développe spontanément; le respect des droits humains tels que nous les entendons aujourd'hui et tels qu'ils sont disponibles pour les constructions qu'en feront les générations à venir n'a rien de naturel. Tout cela demande éducation, explicitation et réflexion. Une des orientations indispensables est notamment le travail sur les concepts qui constituent le cœur de la réflexion démocratique et les débats dont celle-ci est l'objet et l'enjeu.

Quant à la vision du monde qu'appelle une éducation à la citoyenneté, nous insistons sur le double risque d'enfermement et donc sur l'ouverture indispensable : enfermement dans le local, enfermement dans le présent. L'enfermement dans le local a lui-même une double signification, 
géographique et sociale. Ainsi, devons-nous apprendre, nous-mêmes et avec nos élèves et nos étudiants à placer les actions, les initiatives, qui sont le plus souvent locales et limitées, dans une perspective et une signification plus amples. Il y a du global dans le local ; nos groupes d'appartenance sont eux-mêmes inclus dans des groupes plus vastes. Quant au présent, nous avons à y échapper non pas en nous réfugiant dans des visions obsolètes du passé, mais en situant nos actions et nos initiatives dans la durée, entre les passés, les présents et les avenirs possibles. La formation politique est une formation à la liberté, liberté de pensée, liberté d'action, liberté de penser autrement l'avenir que prédéterminé par des contraintes auxquelles nous n'aurions que l'obligation d'obéir, liberté d'action qui invite aussi à introduire des pratiques différentes dans l'univers scolaire.

Cette dimension du temps caractérise aussi l'éducation à la citoyenneté. Celle-ci se déroule, ou devrait se dérouler tout au long de la scolarité et impliquer constamment une formation à l'esprit critique, condition même de la liberté qui vient d'être soulignée (Ethier, Lefrançois \& Audigier, 2018). De nombreux travaux et de nombreuses publications analysent et décrivent des orientations théoriques et pratiques diverses et innovantes. Toutefois, au-delà de cette nécessaire et heureuse diversité, il convient de rester très ferme sur les principes et de ne pas réduire, l'EC à quelques dispositifs et situations principalement dédiés à l'ordre scolaire et social. Cela appelle de la part des formateurs et des enseignants des convictions fortes et assurées. Cela implique aussi une formation des enseignants digne des finalités de l'EC. Une telle formation est trop souvent réduite, voire absente, dans un trop grand nombre d'institutions de formation.

\section{Références bibliographiques}

Audigier, F. (2016). Débat et formation politique: interrogation sur une nouvelle 'norme pédagogique' autour de l'exemple de l'éducation en vue du développement durable. In M. A. Ethier \& E. Mottet (Eds.). Didactiques de l'histoire, de la géographie et de l'éducation à la citoyenneté (129138). Bruxelles: De Boeck.

Audigier, F., Fink, N., Freudiger, N. \& Haeberli, Ph. (Eds.) (2011). L'éducation en vue du développement durable: sciences sociales et élèves en débats. Cabiers de la section des sciences de l'éducation, 130. Genève: Université de Genève.

Boltanski, L. (1990). L'amour et la justice comme compétences. Trois essais de sociologie de l'action. Paris: Métailié.

Bourdieu, P. \& Passeron, J.C. (1970). La reproduction. Eléments pour une théorie du système d'enseignement. Paris: Les éditions de Minuit.

Bruner, J. (2002). Pourquoi nous racontons-nous des histoires? Paris: Retz.

Coleman, J.S. (1966). Equality of Educational Opportunity. Washington, D.C.: GPO.

Crahay, M. (2000). L'école peut-elle être juste et efficace ? Bruxelles: De Boeck.

Debarbieux, E. (1999). Désigner et Punir. In D. Meuret (éd.). La justice du système éducatif (195-209). Bruxelles : De Boeck.

Damasio, A. (2018). L'Ordre étrange des choses. La vie, les sentiments et la fabrique de la culture. Pars: Odile Jacob.

Dewey, J. (1955). Liberté et culture. Paris: Aubier Montaigne.

Dubet, F. (1991). Les lycéens. Paris: Seuil.

Dubet, F. (1999). Sentiments et jugements de justice dans l'expérience scolaire. In D. Meuret (éd.). La justice du système éducatif (177-194). Bruxelles: De Boeck.

Duru-Bellat, M. \& Meuret, D. (2009). Les sentiments de justice à et sur l'école. Bruxelles: De Boeck.

Ethier, M.A., Lefrançois, D. \& Audigier, F. (dir.) (2018). Pensée critique, enseignement de l'bistoire et de la citoyenneté. Louvain-la-Neuve: De Boeck. 
Freinet, C. (1960). L'éducation morale et civique. Paris: Maspéro.

Freinet, C. (1971). Pour l'école du peuple. Paris: Maspéro.

Gosseries, A. \& Meyer, L. (2009). Intergenerational Justice. Oxford: Oxford University Press.

Haeberli, Ph. (2009). Contribution à l'étude d'une compétence dans le domaine de la citoyenneté : l'exemple de la justice dans les conseils de classe de l'école primaire genevoise (Thèse doctorale). Extrait de https://archive-ouverte.unige.ch/unige: 13237

Laplace, C. (2002). Approche clinique des pratiques du conseil d'élèves dans l'École genevoise (1990-2001) (Thèse doctorale).

Lordon, F. (2013). Les affects de la politique. Paris: Seuil.

Merle, P. (2002). L'humiliation des élèves dans l'institution scolaire : contribution à une sociologie des relations maître-élèves, Revue Française de Pédagogie, 139, 31-51.

Prairat, E. (2003). La sanction en éducation. Paris: PUF.

Rawls, J. (1971). Théorie de la justice. Paris: Seuil.

Ricoeur, P. (1983). Temps et récit. Tome 1. Paris: Seuil.

Ricoeur, P. (1990). Soi-même comme un autre. Paris: Seuil.

Vergnioux, A. (2005). Cinq études sur Célestin Freinet. Caen: Presses Universitaires de Caen

Vincent, G., Lahire, B. \& Thin, D. (1993). L'éducation prisonnière de la forme scolaire. Lyon: Presses Universitaires de Lyon.

Walzer, M. (1998). Sphères de justice. Une défense du pluralisme et de l'égalité. Paris: Seuil.

Walzer, M. (2003). Raison et passion. Pour une critique du libéralisme. Paris: Circé.

\section{Información sobre los autores}

Autor: Philippe Haeberli

Institución: Université de Genève

Email: philippe.haeberli@unige.ch

Autor: François Audigier

Institución: Université de Genève

Email: francois.audigier@unige.ch 


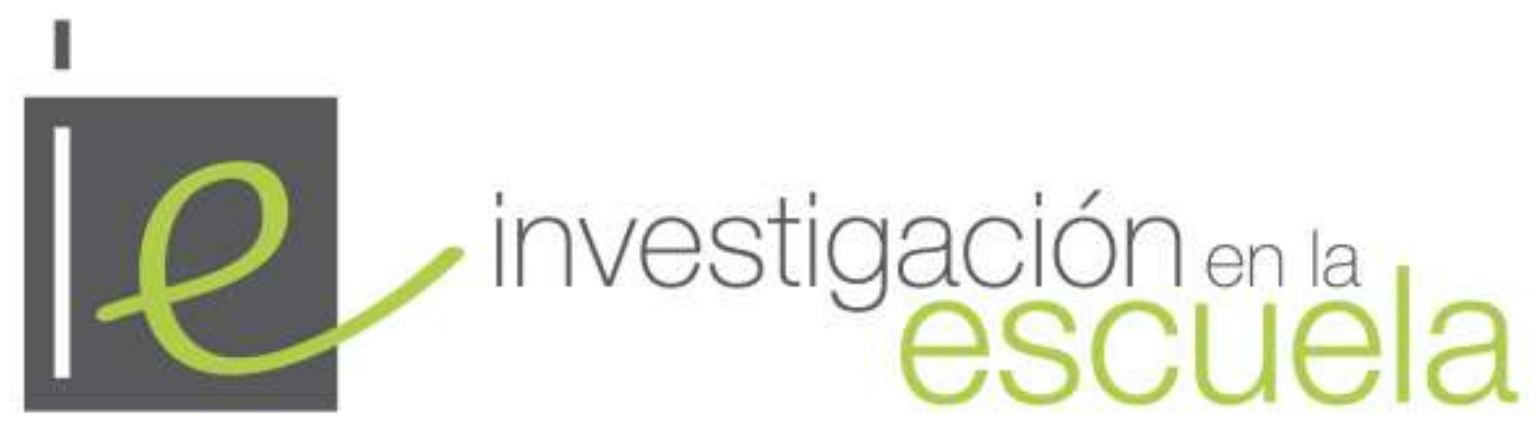

Revista académica evaluada por pares y de acceso abierto

Número 97

30 de abril de 2019

ISSN 2443-9991

\section{(c)}

SOMREFIIGHISRESERVEDLOS/as lectores/as pueden copiar, mostrar, y distribuir este artículo, siempre y cuando se de crédito y atribución al autor/es y a Investigación en la Escuela, se distribuya con propósitos no-comerciales, no se altere o transforme el trabajo original. Más detalles de la licencia de CreativeCommons se encuentran en http://creativecommons.org/licenses/by-nc-sa/3.0 Cualquier otro uso debe ser aprobado en conjunto por el autor/es, o Investigación en la Escuela.

(t) investigacion-en-la-escuela

Contribuya con comentarios y sugerencias en la web de la revista. Por errores y sugerencias contacte a secretaria@investigacionenlaescuela.es

La revista Investigación en la Escuela desde su origen en 1987 hasta su nº 87 (2015) fue editada por Díada Editora. 
